\title{
Higgs boson mass bounds in the presence of a heavy fourth quark family
}

\author{
John Bulava \\ CERN Theory Devision \\ E-mail: john.bulava@cern.ch \\ Philipp Gerhold, Jim Kallarackal, Attila Nagy* \\ Humboldt University Berlin \\ E-mail: philipp_gerholdehotmail.com, \\ Jim.kallarackal@physik.hu-berlin.de, \\ nagy@physik.hu-berlin.de

\section{Karl Jansen} \\ NIC, Desy Zeuthen \\ E-mail: karl.jansen@desy.de
}

\begin{abstract}
We present Higgs boson mass bounds in a lattice regularization allowing thus for non-perturbative investigations. In particular, we employ a lattice modified chiral invariant Higgs-Yukawa model using the overlap operator. We show results for the upper and lower Higgs boson mass bounds in the presence of a heavy mass-degenerate quark doublet with masses ranging up to $700 \mathrm{GeV}$. We perform infinite volume extrapolations in most cases, and examine several values of the lattice cutoff. Furthermore, we argue that the lower Higgs boson mass bound is stable with respect to the addition of higher dimensional operators to the scalar field potential. Our results have severe consequences for the phenomenology of a fourth generation of quarks if a light Higgs boson is discovered at the LHC.
\end{abstract}

The 30th International Symposium on Lattice Field Theory

June 24 - 29, 2012

Cairns, Australia

\footnotetext{
${ }^{*}$ Speaker.
} 


\section{Introduction}

The existence of the standard model Higgs boson is a necessary ingredient for the consistency of the standard model. Although the mass of the Higgs boson cannot be predicted, bounds on the Higgs boson mass can be given. A lower bound can be derived from the requirement of a stable vacuum while an upper bound can be computed from the triviality of the theory which leads to the occurrence of the Landau pole. These mass bounds are usually computed in perturbation theory and indeed, perturbative results for the Higgs boson mass bounds were obtained in the past. However, the perturbative analysis may be questioned, since for the upper bound, the quartic coupling can become strong. The lower bound, on the other hand, may be an artefact of perturbation theory when looking at Higgs field values far away from the minimum of the potential. These concerns motivated a non-perturbative and ab initio lattice field theory investigation of the mass bounds of the Higgs boson in a chirally invariant lattice Higgs-Yukawa model [1,2].

In the lattice approach it is straightforward to determine those mass bounds also for the case that there is a fourth, heavy family of quarks. Such an extension is not excluded a priori and further, a fourth family of fermions offers the possibility of generating sufficient CP violation to fulfil the Sakharov condition to explain electroweak baryogenesis of the early universe [3].

However, CKM4 fits and direct searches already make a naive fourth generation scenario quite improbable [4], especially if the newly discovered particle with a mass around $126 \mathrm{GeV} \mathrm{[5,6]} \mathrm{turns}$ out to be the standard model Higgs boson. In this work we provide additional general constraints on the fourth family quark masses by their effect on the Higgs boson mass bounds.

\section{Model and Implementation}

We study the Higgs-Yukawa sector of the standard model. The field content we consider is a fermion doublets $\Psi$ and a complex scalar doublet $\varphi$. The continuum action in this model is given by:

$$
\begin{aligned}
S^{\mathrm{cont}}[\bar{\psi}, \psi, \varphi]= & \int d^{4} x\left\{\frac{1}{2}\left(\partial_{\mu} \varphi\right)^{\dagger}\left(\partial^{\mu} \varphi\right)+\frac{1}{2} m_{0}^{2} \varphi^{\dagger} \varphi+\lambda_{0}\left(\varphi^{\dagger} \varphi\right)^{2}\right\} \\
& +\int d^{4} x\left\{\bar{t} \not \partial t+\bar{b} \not \partial b+y_{b_{0}} \bar{\psi}_{L} \varphi b_{R}+y_{t_{0}} \bar{\psi}_{L} \tilde{\varphi} t_{R}+\text { h.c. }\right\}, \quad \text { with } \tilde{\varphi}=i \tau_{2} \varphi^{*} .
\end{aligned}
$$

The bare quartic self coupling of the scalar field is given by $\lambda_{0}, m_{0}$ denotes the bare mass of the scalar field and $y_{t_{0}} / b_{0}$ are the Yukawa couplings of the fermion fields. We want to stress, that we do not include any gauge fields in this model neither the gluonic degrees of freedom nor electroweak ones. This is done for computational simplicity and further we expect the effect of the gauge fields to be small for the problems addressed in our work.

We will mostly study the system in the phase with spontaneous symmetry breaking where the scalar field develops a non vanishing vacuum expectation value (vev) and three Goldstone modes emerge. Further we only consider the heaviest fermion doublet, since the dynamics of the scalar field is dominated by the largest Yukawa coupling. 
For the discretized lattice version of the bosonic action we rewrite the scalar doublet as a real four vector $\Phi$. With the reparametrisations

$$
a \varphi=\sqrt{2 \kappa}\left(\begin{array}{c}
\Phi^{2}+i \Phi^{1} \\
\Phi^{0}-i \Phi^{3}
\end{array}\right), \quad \lambda_{0}=\frac{\hat{\lambda}}{4 \kappa^{2}}, \quad a^{2} m_{0}^{2}=\frac{1-2 \hat{\lambda}-8 \kappa}{\kappa}
$$

the bosonic action can then be written in the compact form:

$$
S_{B}[\Phi]=-\kappa \sum_{x, \mu} \Phi_{x}^{\dagger}\left[\Phi_{x+\mu}+\Phi_{x-\mu}\right]+\sum_{x} \Phi_{x}^{\dagger} \Phi_{x}+\hat{\lambda} \sum_{x}\left[\Phi_{x}^{\dagger} \Phi_{x}-1\right]^{2} .
$$

Here $\kappa$ denotes the hopping parameter. The subscripts $x$ and $x \pm \mu$ on the scalar fields denote the scalar field at the space-time point $x$ and $x \pm a \hat{\mu}$ respectively.

For the discretization of the fermions we use the overlap operator $D^{o v}[7,8,9]$ with a Wilson Dirac kernel $D^{W}$ :

$$
D^{o v}=\rho\left\{1+\frac{A}{\sqrt{A^{\dagger} A}}\right\}, \quad A=D^{W}-\rho, \quad D^{W}=\sum_{\mu} \gamma_{\mu} \nabla_{\mu}^{s}-\frac{r}{2} \nabla_{\mu}^{b} \nabla_{\mu}^{f},
$$

with $\nabla_{\mu}^{f, b, s}$ being the forward, backward and symmetrized lattice nearest neighbor difference operators in direction $\mu$. The so-called Wilson parameter $r$ is as usual chosen to be $r=1$. The dimensionless parameter $\rho$ is free to be chosen in the range $0<\rho<2 r$. However, the locality properties of the free overlap operator in case of vanishing gauge couplings are optimal for the case of $\rho=1$ [9], which will therefore be the choice in our work. The fermionic part of the action is then:

$$
S_{F}[\bar{\psi}, \psi, \Phi]=\sum_{x} \bar{\psi}_{x}\left[D^{o v}+P_{+} \phi_{x}^{\dagger} \operatorname{diag}\left(\hat{y}_{t}, \hat{y}_{b}\right) \hat{P}_{+}+P_{-} \operatorname{diag}\left(\hat{y}_{t}, \hat{y}_{b}\right) \phi_{x} \hat{P}_{-}\right] \psi_{x}, \quad \hat{y}_{t / b}=\sqrt{2 \kappa} y_{t / b_{0}} .
$$

This action now obeys an exact global $\mathrm{SU}(2)_{L} \times \mathrm{U}(1)_{Y}$ lattice chiral symmetry with the transformations:

$$
\psi \rightarrow U_{Y} \hat{P}_{+} \psi+U_{Y} \Omega_{L} \hat{P}_{-} \psi, \quad \bar{\psi} \rightarrow \bar{\psi} P_{+} \Omega_{L}^{\dagger} U_{Y}^{\dagger}+\bar{\psi} P_{-} U_{Y}^{\dagger}, \quad \phi \rightarrow U_{Y} \phi \Omega_{L}^{\dagger}, \quad \phi^{\dagger} \rightarrow \Omega_{L} \phi^{\dagger} U_{Y}^{\dagger},
$$

for any $\Omega_{L} \in \mathrm{SU}(2)_{L}$ and $U_{Y} \in \mathrm{U}(1)_{Y}$. The modified chiral projectors are given by:

$$
\hat{P}_{+/-}=\frac{1 \pm \hat{\gamma}^{5}}{2}, \quad \hat{\gamma}^{5}=\gamma^{5}\left(1-\frac{1}{\rho} D^{o v}\right)
$$

Even though in principle different masses for the fermions in the doublet are possible we restrict ourselves to a mass-degenerate doublet in this work. For the implementation we use a polynomial Hybrid Monte Carlo algorithm [10] with various improvements implemented. For further details of the implementation see [11].

To set the scale, i.e. to determine the lattice spacing $a$, we use the phenomenologically known value of the vev of $246 \mathrm{GeV}$. Further we define the cutoff $\Lambda$ as the inverse of the lattice spacing. Since in finite volume a naive average scalar field would vanish without an external field, we define the magnetization $m$ as the average absolute value of the scalar field:

$$
m=\langle|\bar{\Phi}|\rangle, \quad \bar{\Phi}=V^{-1} \sum_{x} \Phi_{x},
$$


with $V$ being the volume of the space time lattice. This approach has the same thermodynamical limit as the application of an external source [12]. The magnetization and the renormalized vev are related as follows:

$$
v_{r}=\frac{m \sqrt{2 \kappa}}{\sqrt{Z_{G}}}, \quad \frac{v_{r}}{a}=246 \mathrm{GeV}, \quad \Lambda=\frac{246 \mathrm{GeV}}{v_{r}} .
$$

The Goldstone and Higgs bosom renormalization constants $Z_{G / H}$ and their masses are computed from the real part of the Goldstone or Higgs boson propagators $G_{G / H}$ respectively:

$$
Z_{G}^{-1}=\left.\frac{d}{d\left(p^{2}\right)} \Re\left(G_{G}^{-1}\left(p^{2}\right)\right)\right|_{p^{2}=-m_{G}^{2}},\left.\quad \Re\left(G_{G / H}^{-1}\left(p^{2}\right)\right)\right|_{p^{2}=-m_{H / G}^{2}}=0 .
$$

The propagators are computed for discrete lattice momenta and fitted according a one loop motivated formula derived in lattice perturbation theory. It may be noted, that this approach especially for the Higgs boson is only valid if the decay width of the particle is small compared to its mass. However, it was shown in a rigorous resonance analysis [13], that at least in the case of a physical top quark mass, the pole of the propagator fully agrees with the resonance mass determined in [13]. Finally, the masses of the fermions $m_{f}$ are computed by means of the time slice correlator

$$
C_{f}(\Delta t)=\frac{1}{L_{t} \cdot L_{s}^{6}} \sum_{t=0}^{L_{t}-1} \sum_{\vec{x}, \vec{y}}\left\langle 2 \Re \operatorname{Tr}\left\{\hat{P}_{L} \psi(t+\Delta t, \vec{x}) \cdot \bar{\psi}(t, \vec{y}) P_{L}\right\}\right\rangle,
$$

which shows a behavior proportional to $\cosh \left(\operatorname{am}_{f}\left(\Delta t-L_{t} / 2\right)\right)$ for large time separations $\Delta t$.

In general we observe rather severe finite volume effects due to the almost massless Goldstone modes, which cause finite volume effects proportional to $L_{s}^{-2}[14,15]$ rather than an exponential falloff with Euclidean time for theories with a mass gap. Some examples for finite volume effects can be found in fig. 1. There, one can see, that for a trustworthy determination of the Higgs boson mass and the vev lattice sizes of up to at least $L_{s}=32$ are necessary.

In addition to the non-perturbative determination we also perform a perturbative analysis by means of the constrained effective potential as described in detail in [11]. Those calculations are performed within the same lattice regularization as the numerical simulations. We employ discrete lattice sums for the loop corrections which are then evaluated numerically. The effective potential $V(\bar{\phi})$ is determined to one loop in the large $N_{f}$ limit with the vev and the Higgs boson mass given by:

$$
\left.\frac{d V(\bar{\phi})}{d \bar{\phi}}\right|_{\bar{\phi}=v}=0 ;\left.\quad \frac{d^{2} V(\bar{\phi})}{d \bar{\phi}^{2}}\right|_{\bar{\phi}=v}=m_{H}^{2}
$$

In order to have a stable vacuum in the scaling regime $(|\bar{\phi}|<0.5)$, we demand the potential to be concave up everywhere in this region. In this perturbative framework we can determine the lower Higgs boson mass bound by finding the lowest possible Higgs boson mass while keeping physical quantities fixed and still fulfil the required stability conditions.

\section{Results}

Let us shortly summarize the strategy to determine mass bounds for the Higgs boson in our analysis. In total we have three free parameters, namely the bare quartic self coupling $\hat{\lambda}$, the 


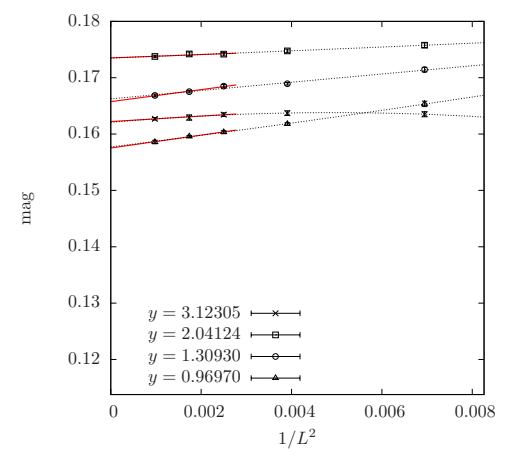

(a) magnetization

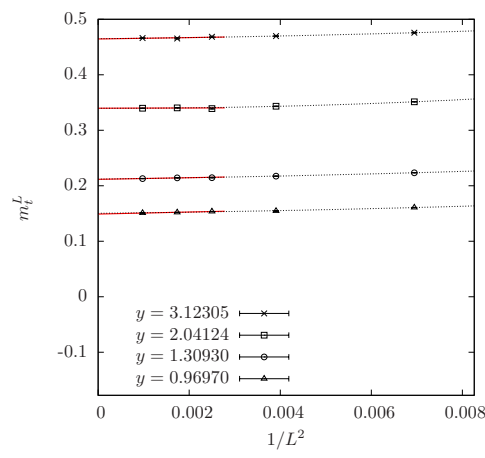

(b) top quark mass

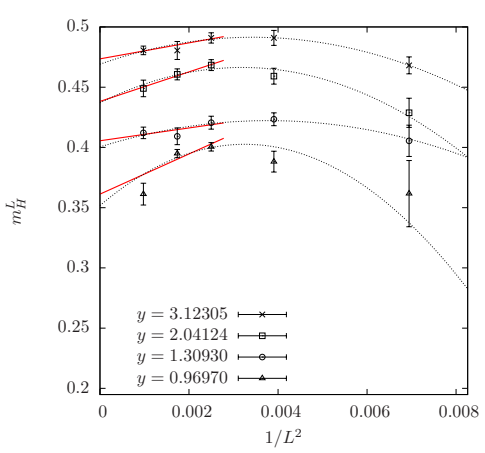

(c) Higgs boson mass

Figure 1: We show finite volume effects for the magnetization, the quark mass and the Higgs boson mass at a cutoff of about $1.5 \mathrm{TeV}$. The data shown correspond to simulations performed at infinite bare quartic coupling and fermion masses in the range $m_{t^{\prime}} \approx 200 \ldots 700 \mathrm{GeV}$. The lattice sizes shown are $L_{s}=12,16,20,24,32$. The red lines indicate linear fits involving only data from computations with $L_{s} \geq 20$ the grey lines show quadratic fits over the whole range of lattice extents.

hopping parameter $\kappa$ and the Yukawa coupling $\hat{y}$. We fix the Yukawa coupling such to obtain the desired mass for the fermions and tune $\kappa$ to retrieve the cutoff at which we want to investigate the mass bounds. Then it can be shown [1,2], that with the cutoff and the fermion mass fixed, the smallest accessible Higgs boson mass is obtained for choosing $\hat{\lambda}=0$ while the Higgs boson mass is the largest for $\hat{\lambda}=\infty$. In earlier works we investigated the cutoff dependence of the Higgs boson mass bounds for the case of a fermion doublet at the physical top quark mass $m_{t} \approx 175 \mathrm{GeV}[1,2]$ and for a very heavy doublet with a mass around $m_{t^{\prime}} \approx 676 \mathrm{GeV}$ [16]. The results can be found in fig. 2. While the upper Higgs boson mass bound is only increased slightly, the lower mass bound for the Higgs boson increased by a factor around 5 when the fermion mass is increased. To obtain a better understanding of the increase in the mass bounds, we investigated those bounds for several fermion masses at a cutoff around $\Lambda \approx 1.5 \mathrm{TeV}$. Those results are shown in fig. 3a. One clearly observes the smooth increase of the lower mass bound of the Higgs boson with increasing quark mass. Our results suggest that a Higgs boson mass of $126 \mathrm{GeV}$ would allow heavy quarks only up to approximately $300 \mathrm{GeV}$.

In fig. 3a we also show the lower Higgs boson mass bound obtained from the perturbative effective potential calculations. It is remarkable, how well the perturbative result agrees qualitatively with the non-perturbative findings up to quark masses of about $700 \mathrm{GeV}$ (corresponding to a Yukawa coupling around 3). This lets us assume, that the model may be perturbative in a wide region of the parameter space. This finding allows us to test perturbatively whether adding a dimension- 6 operator, i.e. a $\lambda_{6} \varphi^{6}$ term, to the bosonic action may have an impact on the lower bound. To this end, we determined the lower bound at a cutoff $\Lambda=2 \mathrm{TeV}$ for various quark masses with and without a dimension- 6 operator at two different couplings $\lambda_{6}$. Those results are shown in fig. $3 \mathrm{~b}$. We clearly see that the additional operator has no visible impact on the lower Higgs boson mass bound. 


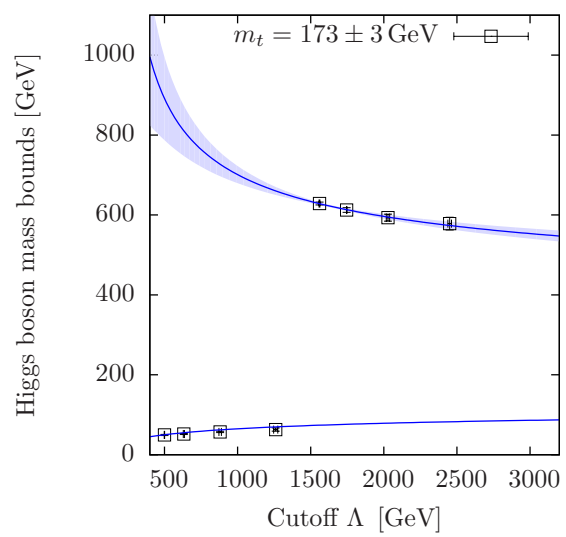

(a) physical top quark mass

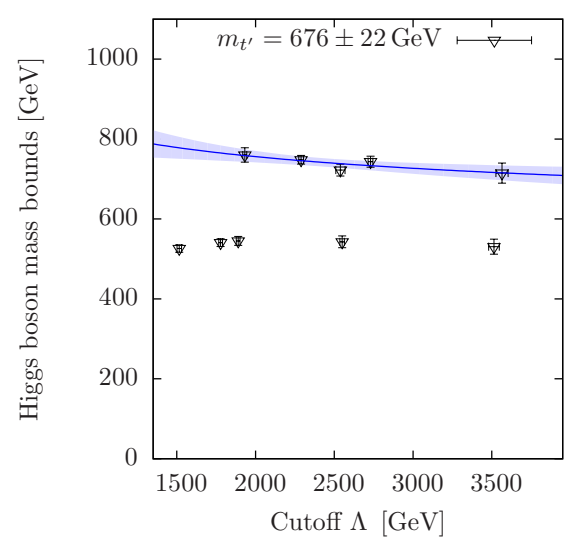

(b) heavy quark double

Figure 2: We show the cutoff dependence of the Higgs boson mass bounds for two masses of a mass degenerate quark doublet. On the left graph we show the data for the physical top quark mass while the right graph corresponds to a very heavy mass around $676 \mathrm{GeV}$.

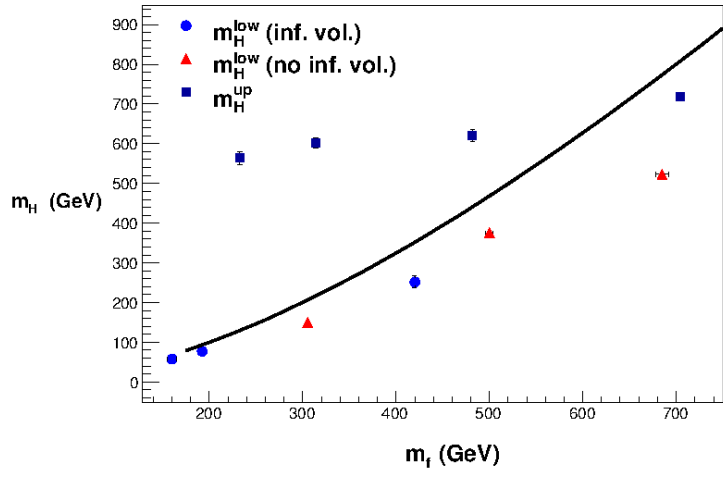

(a) Results at $\Lambda \approx 1.5 \mathrm{TeV}$



(b) Higher dimensional operators

Figure 3: We show Higgs boson mass bounds at fixed cutoff. On the left graph non-perturbative data are shown for the upper and lower mass bound. (For the red triangles the infinite volume extrapolation has not been carried out yet). The data points for the lower bound are compared to findings in a perturbative effective potential calculation. The right-hand plot compares perturbative results for the lower bound with and without a dimension- 6 operator included at a cutoff $\Lambda=2 \mathrm{TeV}$.

\section{Conclusion and Outlook}

We performed a non-perturbative determination of Higgs boson mass bounds in a HiggsYukawa model with a physical top quark mass and a heavy quark doublet. We found that a $126 \mathrm{GeV}$ Standard Model Higgs boson excludes a naive fourth generation of quarks if their mass exceeds $300 \mathrm{GeV}$. Further we gave perturbative arguments, that this bound is stable against the inclusion of higher dimensional operators. However, we plan to addressed this finding also non-perturbatively in the future. Further, our setup allows to test whether a non-degenerate doublet could alter the mass bounds, since a mass splitting of up to $m_{b^{\prime}} / m_{t^{\prime}} \approx 0.9$ is possible [17]. Another interesting direction would be to include an additional scalar field, as suggested in [18], which could have an impact on the Higgs boson mass bound. 


\section{Acknowledgements}

This work was supported by the DFG through the DFG-project Mu932/4-4. The numerical Simulation have been performed at the SGI system HLRN-II at the HLRN supercomputing service in Berlin and Hannover and on the HPC cluster at DESY Zeuthen.

\section{References}

[1] P. Gerhold and K. Jansen. Lower Higgs boson mass bounds from a chirally invariant lattice Higgs-Yukawa model with overlap fermions. JHEP, 0907:025, 2009.

[2] P. Gerhold and K. Jansen. Upper Higgs boson mass bounds from a chirally invariant lattice Higgs-Yukawa model. JHEP, 1004:094, 2010.

[3] B. Holdom, W.S. Hou, T. Hurth, M.L. Mangano, S. Sultansoy, et al. Four Statements about the Fourth Generation. PMC Phys., A3:4, 2009.

[4] O. Eberhardt, G. Herbert, H. Lacker, A. Lenz, A. Menzel, et al. Impact of a Higgs boson at a mass of $126 \mathrm{GeV}$ on the standard model with three and four fermion generations. 2012.

[5] S. Chatrchyan et al. Observation of a new boson at a mass of $125 \mathrm{GeV}$ with the CMS experiment at the LHC. Phys.Lett., B716:30-61, 2012.

[6] G. Aad et al. Observation of a new particle in the search for the Standard Model Higgs boson with the ATLAS detector at the LHC. Phys.Lett., B716:1-29, 2012.

[7] H. Neuberger. Exactly massless quarks on the lattice. Phys.Lett., B417:141-144, 1998.

[8] H. Neuberger. More about exactly massless quarks on the lattice. Phys.Lett., B427:353-355, 1998.

[9] P. Hernandez, K. Jansen, and M. Lüscher. Locality properties of Neuberger's lattice Dirac operator. Nucl.Phys., B552:363-378, 1999.

[10] R. Frezzotti and K. Jansen. A Polynomial hybrid Monte Carlo algorithm. Phys.Lett., B402:328-334, 1997.

[11] P. Gerhold. Upper and lower Higgs boson mass bounds from a chirally invariant lattice Higgs-Yukawa model.

[12] M. Göckeler and H. Leutwyler. Constraint correlation functions in the $\mathrm{O}(\mathrm{N})$ model. Nucl.Phys., B361:392-414, 1991.

[13] Philipp Gerhold, Karl Jansen, and Jim Kallarackal. The Higgs boson resonance width from a chiral Higgs-Yukawa model on the lattice. Phys.Lett., B710:697-702, 2012.

[14] P. Hasenfratz and H. Leutwyler. Goldstone boson related finite size effects in field theory and critical phenomena with O(n) symmetry. Nucl.Phys., B343:241-284, 1990.

[15] A. Hasenfratz, K. Jansen, J. Jersak, H.A. Kastrup, C.B. Lang, et al. Goldstone bosons and finite size effects: A Numerical study of the O(4) model. Nucl.Phys., B356:332-366, 1991.

[16] P. Gerhold, K. Jansen, and J. Kallarackal. Higgs boson mass bounds in the presence of a very heavy fourth quark generation. JHEP, 1101:143, 2011.

[17] A. Denner, S. Dittmaier, A. Muck, G. Passarino, M. Spira, et al. Higgs production and decay with a fourth Standard-Model-like fermion generation. Eur.Phys.J., C72:1992, 2012.

[18] J. Elias-Miro, J. R. Espinosa, G. F. Giudice, H. M. Lee, and Alessandro Strumia. Stabilization of the Electroweak Vacuum by a Scalar Threshold Effect. JHEP, 1206:031, 2012. 